\title{
eJRIEPS
}

Ejournal de la recherche sur l'intervention en éducation physique et sport

35 | 2015

Varia

\section{L'enseignement et l'apprentissage de la tactique en sports collectifs : des précurseurs oubliés aux perspectives actuelles}

\author{
Jean-Francis Gréhaigne
}

\section{(2) OpenEdition}

Édition électronique

URL : http://journals.openedition.org/ejrieps/1660

DOI : 10.4000/ejrieps. 1660

ISSN : 2105-0821

Éditeur

ELLIADD

\section{Référence électronique}

Jean-Francis Gréhaigne, «L'enseignement et l'apprentissage de la tactique en sports collectifs : des précurseurs oubliés aux perspectives actuelles », eJRIEPS [En ligne], 35 | 2015, mis en ligne le 01 avril 2015, consulté le 03 octobre 2019. URL : http://journals.openedition.org/ejrieps/1660 ; DOI : 10.4000/ ejrieps. 1660

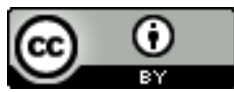

La revue eJRIEPS est mise à disposition selon les termes de la Creative Commons Attribution 4.0 International License. 


\title{
eJRIEPS 35 avril 2015
}

\section{L'enseignement et l'apprentissage de la tactique en sports collectifs : des précurseurs oubliés aux perspectives actuelles}

\author{
Jean-Francis Gréhaigne* \& Luc Nadeau* \\ * Professeur retraité de l'Université de Franche-Comté, Besançon, France \\ ** Professeur de l'Université Laval, Québec, Canada
}

\section{Résumé}

L'objet de cet article est de proposer quelques données historiques et contemporaines sur l'évolution des conceptions de l'enseignement / apprentissage de la tactique en sports collectifs. II tente de mieux décrire et comprendre les enjeux actuels de la recherche sur l'enseignement et l'apprentissage en sports collectifs. A cet effet, un large tour d'horizon de la littérature internationale est effectué pour bien analyser les apports des chercheurs et spécialistes à cette construction.

Mots clés : sport collectif, enseignement, apprentissage, tactique, compréhension.

Un décalage important entre les différents courants de recherche sur l'enseignement et l'apprentissage des sports collectifs semble souvent séparer les conceptions des spécialistes et chercheurs du monde anglophone avec ceux des autres langues. C'est le cas particulièrement dans un certain nombre de pays d'Europe comme la France, le Portugal, l'Espagne ou l'Italie. C'est grâce à des discussions fréquentes avec des collègues de par le monde et à l'occasion des congrès de l'Association Internationales des Ecoles Supérieures d'Education Physique (AIESEP) que nous avons pris conscience de ce décalage dans les perceptions des experts sur les fondements de la recherche dans ce domaine. Bien qu'en utilisant souvent les mêmes mots, les chercheurs de par le monde n'y rattachent ni les mêmes faits, ni les mêmes concepts. Ce constat nous a amenés à reprendre quelques données historiques et contemporaines sur l'évolution de l'apprentissage et des conceptions sur l'enseignement / apprentissage des jeux et sports collectifs (JSC) afin de tenter de mieux comprendre les enjeux actuels de la recherche à propos de ces thèmes. Cet exercice devrait permettre de mieux interpréter l'évolution des courants de recherche concernant l'enseignement des JSC et mieux aider les professeurs d'EPS à comprendre les enjeux des nouvelles méthodes d'enseignement / apprentissage qu'ils peuvent utiliser auprès de leurs élèves. Enfin, nous proposerons une analyse 


\section{eJRIEPS 35 avril 2015}

didactique de l'approche tactique en sport collectif que nous caractérisons comme une approche socio-sémio-constructiviste visant à renouveler les fondements de l'apprentissage des élèves

\section{A propos des débats théoriques sur les modèles d'enseignement des sports collectifs}

Pour nous d'abord, un modèle d'enseignement est une représentation fonctionnelle et simplifiée d'un agencement spécifique d'activités et d'interventions. Ce modèle est basé sur une conception particulière des caractéristiques de l'élève et de l'apprentissage dans un but d'éducation. II constitue un guide pour l'élaboration des contenus du cours, pour le choix des activités ainsi que pour l'animation des interactions entre les élèves et l'enseignant. Ainsi, les fondements de l'enseignement des JSC sont basés habituellement sur des modèles ayant des visées bien différentes. D'une part, certains professeurs d'EPS utilisent un modèle d'enseignement dit traditionnel qui vise à développer les habiletés motrices de base (habiletés techniques) à travers la répétition de gestes. Or, comme nous le verrons, ces modèles ont démontré leur efficacité pour l'apprentissage des habiletés motrices isolées, mais ne permettent pas aux joueurs de mieux comprendre le jeu et de choisir le moment approprié pour exécuter l'action en question. Cet aspect est essentiel dans les JSC où la proximité des coéquipiers et adversaires amènent les joueurs à choisir les meilleures actions possibles leur permettant de marquer des buts et empêcher l'adversaire d'en marquer. À l'opposé, plusieurs modèles basés sur la compréhension du jeu sont maintenant utilisés dans les écoles et nous semblent beaucoup plus appropriés pour faire apprendre les fondements tactiques à la base des JSC. Ces modèles fondés sur la compréhension ont été développés par plusieurs chercheurs de nombreux pays au cours des dernières décennies. Le texte qui suit présente quelques données chronologiques qui permettent de développer une revue de questions élargie sur les études portant sur l'enseignement et l'apprentissage des JCS pour montrer leurs origines (trop souvent oubliées) et pointer les perspectives. Une revue de la littérature comme celle-ci comporte toujours une part de subjectivité et pour ce travail, nous avons choisi de présenter les travaux des auteurs emblématiques concernant ce thème de l'approche tactique. Ce texte s'inspire principalement des travaux de Godbout et Gréhaigne ainsi que des références du TGFU sub interest group en relation avec différentes présentations aux congrès de l'Association pour la Recherche sur l'Intervention en Sport (ARIS) et de l'Association Internationale des Ecoles supérieures d’Education Physique (AIESEP). 


\section{eJRIEPS 35 avril 2015}

\section{Quelques moments de la réflexion tactique en JSC}

La première remise en cause de l'omnipotence du modèle technique et individualiste qui a longtemps été à la base de l'enseignement des jeux et des JSC date principalement du début des années 60 (Dyotte \& Ruel, 1976). À cette époque, la suprématie de l'équipe de hockey sur glace d'Union Soviétique était notable dans les grandes compétitions internationales. Grâce à un jeu d'attaque collectif, basé sur la rapidité et fait de passes avec une remarquable circulation des joueurs qui inter changeaient de positions avec une très grande cohésion, les joueurs de l'ex URSS surpassaient leurs adversaires dans toutes les phases du jeu, même les joueurs professionnels nord américains. La doctrine mise en place alors affirmait que l'on ne peut pas gagner un match en ne jouant que défensivement, à moins d'avoir de la chance. Aussi, l'équipe d’Union Soviétique développa un jeu où les joueurs permutaient continuellement pour mieux confondre les défenses adverses, par opposition au hockey pratiqué à l'époque au Canada, où chacun restait davantage figé dans sa position et ne se déplaçait que d'avant en arrière, avec peu de liberté (Dyotte \& Ruel, 1976). L'entraîneur soviétique, Anatoli Tarasov, prônait un jeu de possession collectif. Au lieu d'envoyer le palet en zone adverse et de partir à sa poursuite ou encore qu'un joueur tente de déjouer, seul, l'opposition, les joueurs soviétiques cherchaient à toujours en garder le contrôle et à progresser par des passes, toujours destinées à un joueur libre et en mouvement. Lorsqu'ils étaient en possession du palet, cette équipe était la première à ne pas prioriser à tout prix une direction de jeu unique vers l'avant à la faveur d'un meilleur contrôle du jeu (Dyotte \& Ruel, 1976).

Par ailleurs, les entraîneurs soviétiques à l'époque ont également introduit l'observation systématique de certaines phases de jeu. Par exemple, à partir d'observations chiffrées avec le pourcentage d'entrées de zone réussies, le pourcentage de palets perdus, les contre-attaques menées par les défenseurs, etc., les entraîneurs et joueurs étaient en mesure de mieux comprendre le jeu des adversaires afin de profiter de leurs faiblesses. Ces observations systématiques étaient beaucoup plus détaillées que les principales statistiques de jeu prises à l'époque par les autres entraîneurs ou spécialistes du hockey sur glace.

\section{Le travail d'avant garde de Frédérich Mahlo et de quelques autres}

Ensuite, le travail de Frédérich Mahlo (1965 et 1966 pour sa publication en allemand) constitue un référent pour les JSC. II établit des relations entre des connaissances 


\section{eJRIEPS 35 avril 2015}

fondamentales et une méthodologie propre aux JSC. « Le fait de résoudre par l'action des problèmes en situation de jeu oblige le plus souvent à ordonner avec discernement la situation problématique et la solution et cela apporte au joueur des connaissances subjectivement nouvelles. Comme l'activité en jeu représente dans son essence même, la solution de nombreux problèmes, apparaissant dans telle ou telle situation, la pensée tactique, en tant que processus intellectuel de cette solution, est une composante indissociable de cette activité » (Mahlo, 1969, p. 29). Au plan des présupposés théoriques, il nous semble justement que Mahlo a été influencé par la théorie de l'activité au sens psychologique telle que développée par Léontiev (1957; 1972 pour un ouvrage en langue française) à la suite de Vygotsky (1933).

\section{CONFIGURATIONS DU JEU}

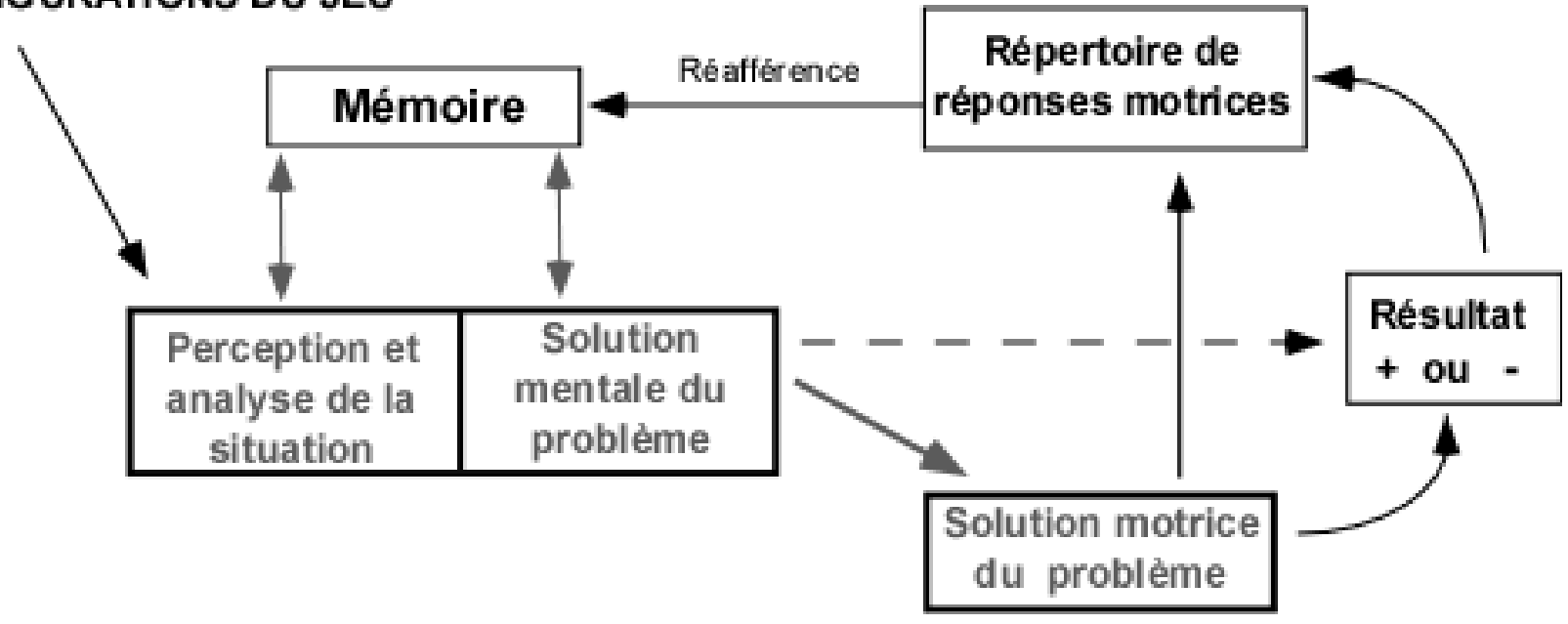

Figure 1. Les phases de l'action de jeu selon Mahlo (1969).

L'analyse de l'action tactique dans les jeux sportifs (figure 1) est donc, en définitive, l'analyse de l'activité en jeu elle-même. Cette attitude vis-à-vis de l'analyse de l'action de jeu fait qu'il existe une interaction entre les fonctions psychiques et la forme réelle ou le type d'activité pour les joueurs. Lorsqu'on analyse l'action tactique, il faut donc partir de ses formes concrètes spécifiques et des signes qu'elle génère.

La solution motrice est en rapport avec le choix des réponses et le moment de leur exécution. Ayant reçu et filtré les informations recueillies sur les configurations du jeu, le joueur doit maintenant décider de l'action appropriée. L'expérience, le répertoire de réponses disponibles, la mémoire, le savoir tactique, les sensations, le jugement et l'activité mentale ont un impact direct sur la détermination du « quoi faire » et du « quand le faire ». De plus, certains facteurs psychologiques tels que l'expérience, la motivation, la place et le statut dans l'équipe ou la confiance influencent aussi cette décision. Le 


\section{eJRIEPS 35 avril 2015}

mouvement ou l'action motrice qui en résulte est par conséquent le fruit d'une décision réfléchie, complexe et volontaire.

Ainsi pour Mahlo, toute activité de jeu est un acte forcément tactique, quel que soit le niveau où se situe le joueur. II consiste à résoudre pratiquement, et dans le respect des règles primaires de l'activité, un grand nombre de problèmes posés par les diverses situations de jeu. Dans cette complexité, nous pouvons, néanmoins, distinguer différents niveaux d'abstraction qui consistent à :

- réfléchir collectivement sur les données concrètes de la situation en liaison étroite avec la perception et le jeu ;

- utiliser la pensée tactique liée au jeu, mais dépassant la situation concrète. Cela opère un rapprochement entre la situation telle qu'elle a été reconnue et des règles, des principes, des réponses. En bref, ce sont la culture et l'expérience au sens de Vygotsky (1933) qui sont constituée par les traces des savoirs techniques sédimentés au fil du développement de l'activité des joueurs pratiquant des sports collectifs ;

- recourir à une pensée tactique abstraite qui n'est pas directement liée à l'acte de jeu, mais qui opère à l'aide de représentations figurées ou de généralisations abstraites pour apporter des réponses aux problèmes posés.

Ce type de travail sera poursuivi en Allemagne par Barth (1978, p. 288) qui, dans un article concernant la stratégie dans les sports d'opposition, parle « d'intelligence de jeu, qui doit permettre une pensée logique, flexible, originale et critique, garantissant l'engagement optimal des habiletés tactiques et permettant des modifications autonomes de l'action selon les circonstances ». Dans l'article de Barth (1980), les termes stratégie et tactique sont déjà bien différenciés et modélisés.

Au Québec, Caron et Pelchat $(1974,1975)$ développeront la notion de «langue de jeu ». Cet objet d'étude est indispensable en vue d'apprendre à jouer en équipe avec la production d'actions en projet. A cet effet, les auteurs proposaient des modèles empiriques et théoriques pour le développement de la tactique en basket-ball et hockey sur glace. Ainsi, la mise en évidence de la « structure du jeu » est un préalable indispensable pour construire la logique du jeu autour de laquelle les actions des joueurs peuvent s'élaborer et s'organiser. L'alternative fondamentale dans les JSC est constituée par la double relation entre continuité du jeu avec les partenaires et opposition avec les adversaires. Pour les joueurs, le développement de la pensée tactique, qui fonctionne par imprégnation et par apprentissage, est nécessaire en vue de résoudre les problèmes posés par les configurations momentanées du jeu. Dans le cas contraire, les joueurs sont amenés à 


\section{eJRIEPS 35 avril 2015}

apprendre schématiquement voire mécaniquement et donc à jouer de même. La réflexion sur la nécessité de combiner les données du jeu et l'exigence de l'auto-information devient primordiale dans les situations d'apprentissage où l'information provient de l'extérieur (les pairs, l'enseignant ou l'entraîneur).

\section{L'évolution des théorisations et des conceptions en France}

En France, les années 1965-1969 marquent un tournant dans la réflexion sur l'enseignement des formes de jeux à l'école.

\subsection{Les pionniers de l'innovation pédagogique}

Tout d'abord, le colloque de Vichy sur l'enseignement des JSC (1964-65) est organisé autour de 3 thèmes: les principes communs à tous les Jeux Sportifs; la formation du joueur depuis l'initiation jusqu'à la compétition ; la place du maître dans l'apprentissage de telle ou telle spécialité. Une liaison théorie / pratique est proposée avec une organisation de la classe (équipes premières et réserves), structurée en club A et B avec un calendrier (progression en vue de compétition).

La détermination des thèmes de jeu va du simple au complexe (systématique des JSC) et du général au particulier. La méthode préconisée définit le rôle de l'entraînement avec une pédagogie adaptée pour tous les élèves. Puis, les écrits relatifs au stage de l'AEEPS (Amicale des Anciens Élèves de l'ENSEPS, 1966) nous paraissent constituer un autre support pour analyser les conceptions dominantes du moment. A cette époque, trois problèmes majeurs étaient abordés sous un angle nouveau : l'acte moteur, le rapport de forces entre les équipes et les modifications à envisager dans le comportement des joueurs.

L'acte moteur ne doit pas être pris isolément, mais compris dans un ensemble dans lequel les données perceptives jouent un rôle majeur. Le match, conçu comme un rapport de forces, doit être analysé dans une perspective dynamique afin d'identifier la structure sur laquelle repose l'organisation de l'équipe. Le joueur devient donc l'élément d'un ensemble structuré permettant la réalisation d'un objectif. L'apprentissage consiste ainsi à modifier l'organisation sensori-motrice du pratiquant à partir d'une intériorisation par celui-ci de la structure de la situation en relation avec l'expérience qui se construit. L'entraînement perd son aspect cumulatif et surtout s'appuie sur des processus cognitifs : perception et reconnaissance de signaux, intériorisation de structures, etc. La progression dans l'apprentissage devient «organique », c'est-à-dire qu'elle prend en compte l'histoire originale vécue par le joueur intégré dans une équipe donnée. Cette équipe est elle-même 


\section{eJRIEPS 35 avril 2015}

confrontée à d'autres équipes dans le cadre d'une sorte de championnat. Les contenus ne sont plus définis a priori, mais sont élaborés à partir d'une analyse par le professeur des différents matchs.

A la même époque, les travaux de Robert Mérand (1989) et de la Fédération Sportive et Gymnique du Travail au sein de son Conseil Pédagogique et Scientifique s'inscrivent dans une perspective originale et ambitieuse : construire des savoirs de la pratique enrichis par un apport de connaissances scientifiques intégrées à la motricité (Vandevelde, 2006). La publication de 1974 à 1978 de mémentos à propos des JSC (par exemple Marsenach \& Druenne, 1974) où les jeux à effectif réduit en situation d'affrontement sont systématiquement utilisés, constitue une évolution importante de la théorisation sur les jeux. Mérand (1977) conceptualise l'idée fondamentale qui organise l'essentiel du renouvellement de l'épistémologie scolaire des JSC en écrivant "l'approche empirique des caractéristiques de l'activité adaptative en situation de jeu, c'est-à-dire lorsque le joueur est confronté aux contraintes et aux possibles du règlement du jeu et du rapport de forces» (p.11). La mise au point d'outils d'observation pertinents tel «l'espace de jeu effectif » constitue un autre point fort de ce travail.

\subsection{Les pionniers de la recherche}

Par ailleurs, Teodorescu (1965) vise à mettre en évidence des principes pour l'étude de la tactique commune aux jeux sportifs collectifs. L'auteur se consacre au fonctionnement interne de l'équipe à l'aide d'analyses (techniques, tactiques....) très précises. Les organisations offensives et défensives sont caractérisées par « des bases, des principes, des facteurs " qui constituent un inventaire très complet de l'ensemble des éléments à prendre en compte pour le bon fonctionnement d'une équipe. Même si ce type d'inventaire reste parfois formel, il concourt à une meilleure compréhension de la structure d'une équipe et des modalités de l'affrontement en soulignant la nécessaire coordination réciproque des actions individuelles et collectives. L'autre contribution de cette approche, par rapport aux méthodes d'enseignement traditionnelles basées sur le découpage en morceaux élémentaires des aspects gestuels chers au technicisme, a consisté à changer la conception d'une équipe. Le groupe de joueurs n'est plus la somme des individus qui la composent, mais un ensemble structuré et dynamique en vue de la réalisation d'un projet commun. II doit exister une coordination réciproque des actions individuelles et collectives à partir de principes généraux pour mieux comprendre et organiser le jeu. Enfin, Teodorescu (1977) montre aussi l'importance de la notion de plan de jeu (préparation 


\section{eJRIEPS 35 avril 2015}

directe de la compétition) et présente une pédagogie instructive où l'assimilation des données repose sur la répétition.

Pour Deleplace $(1966,1979)$ et Villepreux (1987) au rugby, techniques et tactiques collectives de base doivent être approfondies en même temps dans leur rapport de réciprocité. Un sport collectif ne s'enseigne pas par chapitres successifs, qu'il suffirait d'accumuler les uns après les autres dans le temps, pour aboutir au jeu complet. II faut tout au long de la formation conserver le jeu réel, source de l'intérêt en même temps que champ d'expérience... Pour l'enseignement, la solution, préconisée par ces pionniers, réside dans l'utilisation de jeux à effectif réduit... Enfin, la clef de voûte des problèmes dans les JSC réside toujours dans une vue claire des liens vivants du jeu, qui sont le produit des éléments objectifs suivants : les courses des attaquants et des défenseurs avec leurs directions et leurs changements de direction, leurs vitesses et leurs changements de vitesse (changement de rythme) ; la vitesse, la direction et le sens du déplacement de la balle. Pour chercher à saisir la vie du mouvement dans sa totalité, il faut donc appréhender ensemble le mouvement du ballon et le mouvement des joueurs dans une réciprocité nécessaire des deux équipes antagonistes. Nous présentons (Figure 2) les éléments permettant de caractériser ces liens inhérents à l'opposition en rugby.

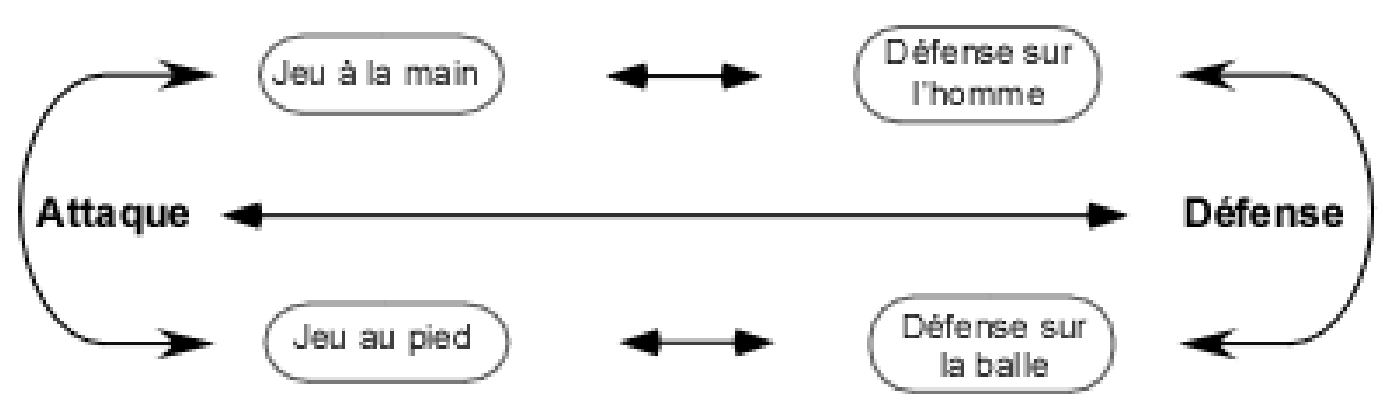

Figure 2. Les liens d'opposition en rugby selon Deleplace (1979).

La gestion par les joueurs de ces liens d'opposition repose sur une prise d'information pertinente des signaux fournis par le jeu. Cela nécessite aussi une compréhension commune et des comportements variés et adaptables permettant la suppléance dans les rôles provisoires en fonction du contexte situationnel. Dans cette optique, les prises d'information constituent le fondement de la disponibilité motrice et tactique du joueur. Cette nécessaire « conscientisation » permet au joueur d'orienter le jeu en possédant un schéma abstrait qui rend compte, de façon opératoire au niveau mental, de la logique d'action correspondant à la situation évolutive particulière à laquelle se rapporte la configuration du jeu en cours. Cela se réalise de façon opératoire, c'est-à-dire d'une façon 


\section{eJRIEPS 35 avril 2015}

qui permet au joueur d'agencer les éléments du jeu. II le fait particulièrement grâce à la possibilité d'anticipation mentale dans l'élaboration d'une suite de décisions au fil de l'action.

A la suite du travail de Deleplace, la pédagogie des modèles de décision tactique (Bouthier, 1986, 1988 ; David, 1984 ; Diaz, 1983 ; Reitchess, 1983 ; Stein, 1981...) «postule que l'intervention des processus cognitifs est décisive dans l'orientation et le contrôle moteur des actions. Elle suppose que la présentation des repères perceptifs significatifs et des principes rationnels de choix tactiques organise de façon majorante les effets du passage à l'acte, y compris en termes de qualité de l'exécution » (Bouthier, 1986, p. 85). Ces résultats d'études montrent néanmoins un réel intérêt pour l'étude de ces problèmes que représentent les processus cognitifs mis en œuvre par les joueurs pendant le jeu. Pour illustrer les travaux effectués autour de la pédagogie des modèles de décisions tactiques (PMDT), nous nous appuierons sur les recherches effectuées par Bouthier (1984). L'idée centrale de ce modèle didactique repose sur l'hypothèse que la présentation des informations essentielles et relatives à l'orientation tactique des actions se produit en situation de deux contre deux. Elles sont ensuite mises en oeuvre dans des unités tactiques relativement isolables du jeu. Cette méthode donne de meilleurs résultats sur l'apprentissage et les performances des joueurs que les deux autres méthodes pédagogiques à la base de l'étude de Bouthier (pédagogie des modèles d'exécution qui repose sur l'apprentissage par le joueur de solutions efficaces produites par les experts, et pédagogie des modèles auto-adaptatifs, s'appuyant sur des variations judicieuses d'aménagement du milieu pour faire découvrir les solutions et développer les habiletés (Lenzen, Poussin, Deriaz, Dénervaud, \& Cordoba, 2010). Sans pouvoir apporter de preuve définitive de ce constat, cette étude suggère que la PMDT comporte une légère supériorité.

De leur côté, Méot et Plumereau (1979, p. 59) signalent que « l'enseignement d'un jeu sportif collectif ne peut être réduit à un apprentissage (par imitation et répétition) d'une gamme de gestes techniques, comme une « série de réponses » correspondant à une série de «situations problèmes". La perspective d'acquérir une attitude de décision permet au joueur d'opérer un choix pour agir dans une situation déterminée, entre autres, par les paramètres suivants :

- la situation topographique du ballon dans l'espace proche ;

- les éléments corporels intervenant sur le ballon ;

- les distances avec les autres joueurs (partenaires, adversaires). 


\section{eJRIEPS 35 avril 2015}

Ici, l'activité perceptive et décisionnelle devient première. On constate que l'intérêt est déjà sur des aspects de tactique individuelle. Méot (1982) ajoute que la formation doit consacrer du temps au développement de l'aptitude à observer fidèlement et méthodiquement, car l'absence de données fiables sur la réalité du jeu entraîne une connaissance empirique éphémère. Ainsi, l'ouvrage de l'Amicale des Enseignants d'Éducation Physique (1984) propose un bilan de dix ans des stages de sports collectifs à Montpellier et en conclut que chacun est maintenant persuadé que la rénovation de l'enseignement des JSC passe par :

-une place privilégiée faite à la réflexion sur les contenus d'enseignement ;

-la mise en œuvre d'une évaluation en cohérence avec l'évolution des pratiques pédagogiques.

II est souligné que la pédagogie ne doit plus viser exclusivement le développement d'habiletés spécifiques, mais viser le développement des capacités et compétences porteuses de potentialité de transfert. Dans le jeu, l'élaboration de réponses nouvelles dépend de la pression temporelle à l'intérieur d'un match (vitesse d'élaboration des réponses). II faut donc jouer «avec » et «sur » le temps dont on dispose pour apprendre.

\subsection{Des aspects institutionnels}

Enfin, au plan plus général et de façon concomitante aux travaux des précurseurs en France, la rénovation des programmes en 67 mettait en perspective un changement de paradigme dans l'enseignement des sports collectifs. En effet, les Instructions Officielles françaises d'éducation physique (IO) qui, en 1967, introduisaient, de fait, les activités physiques et sportives à l'école. Ces instructions débutaient par ces mots : «l'organisation des activités physiques et sportives, en milieu scolaire et universitaire, et l'insertion du sport, phénomène social et culturel, dans les programmes d'enseignement, soulèvent un certain nombre de problèmes pédagogiques ".

Aussi me paraît-il indispensable de préciser la notion d'éducation physique et de situer cette discipline parmi les autres et de définir son domaine et d'introduire, entre les activités physiques et sportives qui en constituent la matière, une cohérence qui est le préalable indispensable à l'élaboration d'un programme" (Ministère de la Jeunesse et des Sports : Ministère de I 'Éducation Nationale, 1967, p. 1).

Dans cet ensemble, la place des activités sportives est prépondérante. Dans la majorité des cas, la compétition constitue une excellente motivation et le meilleur moyen de contrôler les résultats obtenus. Avec le programme détaillé des activités concernant les JSC, il est précisé que la connaissance des niveaux de jeu par le professeur, pouvant s'appuyer sur l'observation mutuelle entre équipes, doit permettre de constater les 


\section{eJRIEPS 35 avril 2015}

insuffisances et de choisir les exercices techniques ou tactiques adaptés aux possibilités des élèves, aux progrès qu'ils sont susceptibles d'accomplir et au niveau de jeu qu'ils peuvent atteindre. On est bien dans la même perspective de ce que l'on a appelé ensuite dans le monde anglophone $\rightarrow$ à la suite de Siedentop (1983) «Sport Education » et qui a fourni un cadre curriculaire pour un enseignement adapté des activités physiques et sportives, sans leur faire perdre leur sens, en vue d'atteindre les objectifs fixés par le programme de l'école. Pour plus de précision sur ce thème, on peut consulter « Sport Education : Une orientation curriculaire nord-américaine » de Amade-Escot (1997).

Par ailleurs, concernant plus spécifiquement le football, Worthington (1974) présente une modélisation basée sur celle initialement développée par Wade (1967). La force concrète de ce modèle réside dans l'idée simple de diminuer le nombre de joueurs sur le jeu et de n'utiliser que six rôles ou positions de jeu, à savoir trois en attaque (attaquant premier, deuxième et troisième attaquant) et trois en défense (le défenseur premier, deuxième et troisième défenseur), pour fournir une compréhension pratique des principes de jeu. Dans ce modèle, les rôles de premier attaquant, deuxième attaquant et troisième sont liés aux principes respectifs de pénétration, de profondeur offensive et de mobilité. D'autre part, les rôles de premier, deuxième et troisième défenseur sont liés aux principes respectifs d'empêcher la pénétration, de construire la profondeur défensive et de maintenir l'équilibre défensif.

Dans sa thèse, avec le dessein de rendre compte plus objectivement des stratégies utilisées dans une rencontre de football, Wilkinson (1978) remarque que la plupart des publications reposent souvent sur l'intuition des entraîneurs à succès. Elles sont teintées de subjectivité et très fréquemment d'une valeur scientifique modeste. Cela le conduit à définir une nouvelle discipline d'enseignement de la stratégie sportive en « faisant appel à la théorie générale des systèmes, à l'utilisation de modèles cybernétiques, à la théorie de l'information, à la théorie mathématique des jeux, à des modèles statistiques et à la connaissance individuelle de la discipline sportive considérée» (p. 27). Cette nouvelle discipline permet d'obtenir des données fiables sur le jeu des joueurs à partir de modèles connus. Dans un deuxième temps, Wilkinson s'attache à définir les composantes de la stratégie sportive. II identifie un grand nombre d'observables concernant le football (Gréhaigne, 1989) et montre que, même avec des moyens modestes, une analyse peut être réalisée et fournir des informations sensiblement plus précises que celles données dans la plupart des cas. Celles-ci ont une signification dans deux directions : d'une part, on peut développer des stratégies à long terme à partir de régularités individuelles pour son 


\section{eJRIEPS 35 avril 2015}

équipe ; et d'autre part, on peut obtenir des informations précises grâce à l'analyse de l'adversaire. Malheureusement, faute d'un modèle intégrateur de ces observables, ce travail n'aboutit pas à des propositions didactiques.

\section{Les années 80 et le « big bang » de l'enseignement pour la compréhension ${ }^{1}$ chez les anglophones}

Les années 1982 / 84 apportent un certain nombre de développements au niveau de l'évolution de la recherche et de la didactique des JSC. Tout d'abord, en France, un nouveau texte concernant l'évaluation et la notation de l'éducation physique au baccalauréat remplace les barèmes nationaux en athlétisme, gymnastique, natation par une évaluation dans trois activités physiques et sportives enseignées 12 semaines dans l'établissement. Ceci entraîne une profonde réflexion et amène un colloque sur l'évaluation en EPS (SNEP, 1984) qui fera date. A la même époque, I'Université de Loughborough publie un premier document sur l'approche par des jeux visant la compréhension (Bunker et Thorpe, 1982). Avec très peu de références théoriques, excepté une vague référence à Wade (1967), une réflexion sur la didactique et la pédagogie des JSC est proposée. Les différents thèmes abordés concernent les classifications des jeux, les règles, l'approche par le jeu et la compréhension. Ils proposent un modèle que nous présentons à la figure 3.

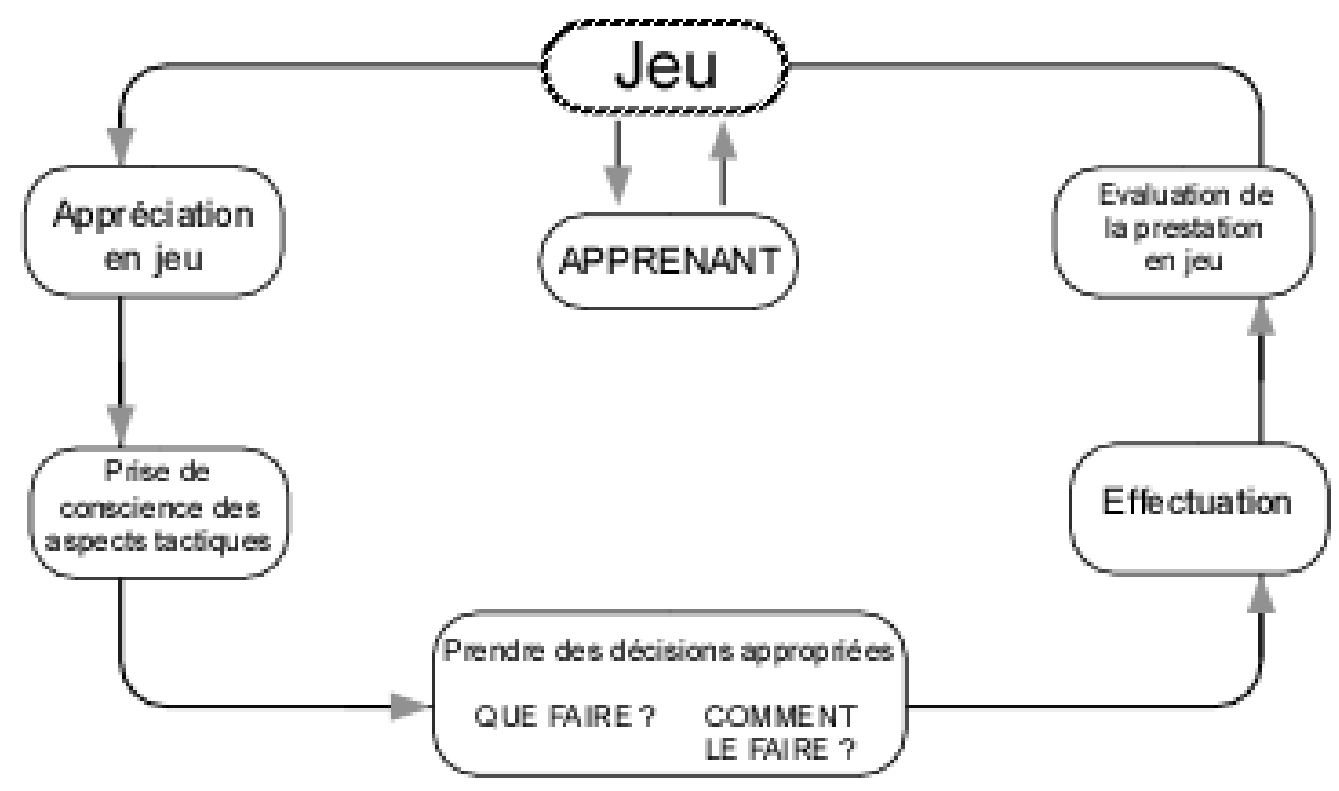

Figure 3. Modèle de Bunker et Thorpe (1982) pour l'enseignement des JSC.

Bien que restant peu testée en termes d'études scientifiques au moment de sa parution, la force de «l'approche l'enseignement du jeu pour la compréhension » de Bunker et Thorpe

\footnotetext{
${ }^{1}$ Traduction littérale en français de Teaching Games for Understanding.
} 


\section{eJRIEPS 35 avril 2015}

(1982) est de mettre l'accent sur la tactique consciente et les procédures de prise de décision avant la sélection des habiletés motrices et leur exécution. Souvent, ce qui discrimine les experts des débutants est leur capacité à prendre les décisions appropriées au regard du jeu (Thomas, French, \& Humphries 1986). Ainsi, «Game sense (Den Duyn, 1997) est une approche qui développe un sens plus large de l'activité physique, car cette approche se concentre sur le développement de la pensée des joueurs par la résolution de problèmes en utilisant des JSC. L'approche «Game Sense » est une variante du modèle de Bunker et Thorpe, et cette approche s'est développée en Australie à la suite d'une visite effectuée par Rod Thorpe en 1996. L'objectif de l'approche est de placer le participant dans des situations où la prise de décision et la résolution de problèmes sont au cœur de la réussite. La stratégie centrale pour l'enseignement ou l'entraînement en utilisant l'approche «game sense » est l'utilisation de questions destinées aux participants pour stimuler la réflexion sur le jeu. Les jeux sont arrêtés à intervalles réguliers et les participants sont mis au défi de réfléchir sur leur participation afin de faire évoluer le jeu.

Le problème récurrent à ces dernières approches fondées sur la réflexion sur le jeu reste cependant la façon de questionner les élèves : où, quand et comment ? En outre, ces questions sont-elles ouvertes ou fermées ? Sont-elles utilisées en permettant des débats uniquement entre élèves ou encore avec le professeur qui pose les questions et apporte les réponses ? L'approche sera ainsi centrée sur les l'élèves, sur la matière enseignée ou encore sur le maître, en fonction des choix effectués.

Dans leur affirmation, Kirk et MacPhail (2002) semblent méconnaître les avancées de la théorisation sur la tactique dans l'enseignement des jeux sportifs collectifs telle qu'initiée par l'école soviétique, poursuivie par Malho et reprise en France dans le domaine de la réflexion didactique en EPS.

Bunker and Thorpe (1982) first proposed Teaching Games for Understanding (TGfU) in 1982 as an alternative to traditional, technique-led approaches to games teaching and learning. Since then, TGfU has attracted widespread attention from teachers, coaches, and researchers (Rink, French, \& Tjeerdsma, 1996). While there have been developments of the Bunker-Thorpe approach in the work of researchers such as Griffin, Oslin, and Mitchell (1997), and Gréhaigne and Godbout (1995), there have been no attempts to revise the Bunker-Thorpe model itself (p. 177).

Parfois, on a l'impression que ce qui n'est pas connu dans le monde anglophone n'existe pas...

\section{L'évolution de part le monde}




\section{eJRIEPS 35 avril 2015}

A partir de 1985, les Américains s'intéressent de nouveau à ce type de problème avec une série de publications qui tentent de montrer la validité d'un enseignement par le jeu avec les prises de décision comme élément central (voir Tableau I).

French et Thomas (1987) ont ainsi trouvé que les jeunes joueurs de basket apprenaient à prendre des décisions justes plus rapidement dans le contexte du jeu que par l'apprentissage d'habiletés techniques. Dans leur recherche, un léger progrès dans les habiletés motrices était observé tout au long de la saison mais au moment du test, rien de significatif ne fut trouvé pour l'effectuation en jeu. French et Thomas (1987) soutiennent que ceci était dû à la quantité de pratique consacrée aux stratégies (au sens américain du terme c'est-à-dire sans différenciation entre stratégie et tactique) plutôt qu'au développement des habiletés motrices du basket-ball.

Lawton (1989) a de son côté travaillé avec des joueurs de badminton de 12 à 13 ans ayant eu une approche combinant la technique et la compréhension. La durée de l'étude de Lawton fut de six semaines. Elle révéla qu'il n'y avait pas de différences significatives pour les connaissances des élèves en badminton dans le temps ou en fonction des approches. Lawton a émis l'hypothèse que l'échec à trouver des progrès significatifs sur les connaissances au post-test pouvait provenir du fait que le pré-test surestimait le niveau initial des sujets sur l'appréciation tactique, bien que les élèves n'aient aucune expérience préalable du badminton.

Turner et Martinek (1992) quant à eux ont préconisé une période expérimentale plus longue que les six semaines de l'étude de Lawton ce qui, d'après eux, aurait peut-être produit des éléments plus stricts pour différencier l'approche jouée de l'approche technique ainsi que leurs effets sur les connaissances des élèves. Gréhaigne (1994) montrera qu'après 6 à 7 leçons, des différences significatives apparaissent en faveur de l'approche tactique.

Tableau I. Quelques travaux américains sur l'apprentissage par le jeu.

\begin{tabular}{|c|c|c|c|c|}
\hline Auteurs & \begin{tabular}{|l|} 
French \& Thomas, \\
1987
\end{tabular} & Lawton, 1989 & \begin{tabular}{|lr} 
Turner & $\&$ \\
Martinek, & 1992 \\
\end{tabular} & Turner, 1993 \\
\hline Activités supports & Basket-ball & Badminton & Hockey / gazon & Hockey / gazon \\
\hline Population & Élémentaire & \begin{tabular}{|l} 
Secondaire \\
\end{tabular} & Secondaire & Élémentaire \\
\hline Durée & Un an & \begin{tabular}{|ll}
6 & semaines \\
$(1 \mathrm{~h})$ & \\
\end{tabular} & 6 semaines & Un semestre \\
\hline Dispositif & $\begin{array}{l}\text { Expt / Novice } \\
\text { Test }\end{array}$ & $\begin{array}{l}3 \text { groupes: Jeu } \\
\text { / Tech. } \\
\text { Contrôle }\end{array}$ & \begin{tabular}{|l|}
$4 \times 4$ \\
Fiche \\
Parcours \\
\end{tabular} & $4 \times 4$ \\
\hline Éléments & Connaissances, & Connaissances & Testent & Connaissances \\
\hline
\end{tabular}


eJRIEPS 35 avril 2015

\begin{tabular}{|l|l|l|l|l|}
\hline analysés & $\begin{array}{l}\text { Compétences } \\
\text { motrices } \\
\text { Prise de décision }\end{array}$ & $\begin{array}{l}\text { déclaratives et } \\
\text { procédurales }\end{array}$ & $\begin{array}{l}\text { approches des } \\
\text { sports collectifs }\end{array}$ & $\begin{array}{l}\text { déclaratives et } \\
\text { procédurales }\end{array}$ \\
\hline Résultats & $\begin{array}{l}\text { Effet sur les } \\
\text { connaissances }\end{array}$ & $\begin{array}{l}\text { Pas de résultats } \\
\text { significatifs }\end{array}$ & $\begin{array}{l}\text { Pas de résultats } \\
\text { significatifs }\end{array}$ & $\begin{array}{l}\text { Effets sur les } \\
\text { connaissances } \\
\text { déclaratives }\end{array}$ \\
\hline
\end{tabular}

Comme pour la pédagogie des «modèles de décision tactique », Riley et Roberton (1981) ont montré que plus les joueurs pratiquent les JSC, plus ils sont capables de s'adapter au jeu. Ils postulent que si une trop grande place est faite au développement des habiletés motrices, ceci construira par la suite des individus qui seront forts au plan technique, mais incapables d'utiliser correctement les stratégies (toujours au sens américain de l'époque) de jeu. L'intégration et l'utilisation des instructions stratégiques et techniques au temps juste doivent avoir un impact sur la performance.

Une autre étude menée par French et Thomas (1987), et qui s'étalait cette fois sur une saison entière, a trouvé un effet principal significatif relatif au temps nécessaire en vue d'un apprentissage, pour l'ensemble des élèves, aussi bien experts que novices. En parallèle, les résultats du groupe contrôle restaient constants du pré-test au post-test. Ils en ont déduit qu'une base de connaissances déclaratives est nécessaire pour le développement des connaissances procédurales et que les enfants qui sont tous débutants manquent souvent d'une somme suffisante de ces deux sortes de connaissances. A la fin de leur expérience, les élèves étaient encore novices en termes de connaissances de base. En outre, Thomas, French, Thomas et Gallagher (1988) soutiennent que "le développement des connaissances procédurales, nécessaires pour prendre des décisions correctes dans le jeu, prend un temps considérable et de longues heures de pratique" (p. 186).

Mac Pherson et French (1991) indiquent que de débuter l'apprentissage par la technique ou par le jeu doit avoir un impact sur les connaissances et sur le jeu. Ils indiquent aussi que les connaissances (déclaratives) des élèves et les prises de décisions dans le jeu augmentent de façon spectaculaire quand l'approche fondée sur le jeu est utilisée quasi exclusivement en premier.

Concernant le problème général des connaissances mises en jeu, les résultats des études restent contradictoires et devraient faire l'objet dans l'avenir de travaux plus approfondis. Les différences constatées entre les résultats dans la littérature peuvent être expliquées par l'âge des sujets et les variations dans les modes d'enseignement (Turner \& Martinek, 1992). Les études de Mac Pherson et French ont porté sur des joueurs de tennis adultes 


\section{eJRIEPS 35 avril 2015}

et débutants et non pas sur des élèves de sixième. Les étudiants participant aux études de Mac Pherson et French avaient aussi à faire des lectures obligatoires sur le jeu pendant leurs activités à l'université en supplément à l'apprentissage. Cette pratique pédagogique ne faisait pas partie de notre étude à l'époque.

Dans une étude similaire relative au badminton, Rink, French et Werner (1991) examinèrent les effets de trois traitements différents (approche tactique, développement des habiletés techniques et une combinaison entre les deux) sur la capacité de jeu de joueurs débutants (étudiants de 9ième, soit environ 14-15 ans). Ils n'ont trouvé aucune différence significative. Par contre, la relation entre les habiletés motrices et la tactique individuelle en jeu au post-test confirme les recherches de French et Thomas (1987). Leur étude sur le basket-ball indique que le tir et la conduite de balle sont reliés aux composantes du jeu et à l'effectuation. La relation entre une habileté technique comme le tir et les actions tactiques individuelles en jeu indique que les élèves sont plus performants dans les situations de jeu à cette étape. A ce propos, Anderson (1982) et Chi et Rees (1983) suggèrent que l'acquisition des connaissances déclaratives constitue les fondements pour un bon développement de connaissances procédurales et permettent aux élèves de savoir quoi faire dans les situations spécifiques de jeu. Toutefois, Mac Pherson et French (1991) pensent que les sujets peuvent sélectionner une certaine réponse dans un contexte de jeu donné et ainsi montrer des procédures d'exécution correctes, sans nécessairement en connaître la raison.

Enfin dans ce genre d'études, les résultats sont rarement statistiquement significatifs car la mesure des apprentissages et de la performance des joueurs est assez complexe. Le petit nombre de sujets et la dispersion des réponses expliquent aussi ce fait. On peut identifier seulement des tendances d'évolution comme bien souvent dans ce genre de recherche de terrain. Néanmoins, cette catégorie d'expérimentation devrait permettre aux enseignants de se faire une idée sur la méthode d'enseignement qu'ils peuvent choisir dans les JSC ainsi que de leurs effets sur les performances des élèves. Cela peut les aider à répondre aux questions du type: "Vaut-il mieux enseigner l'aspect technique ou tactique du sport collectif ; Quelle place accorder à la technique ? etc. 


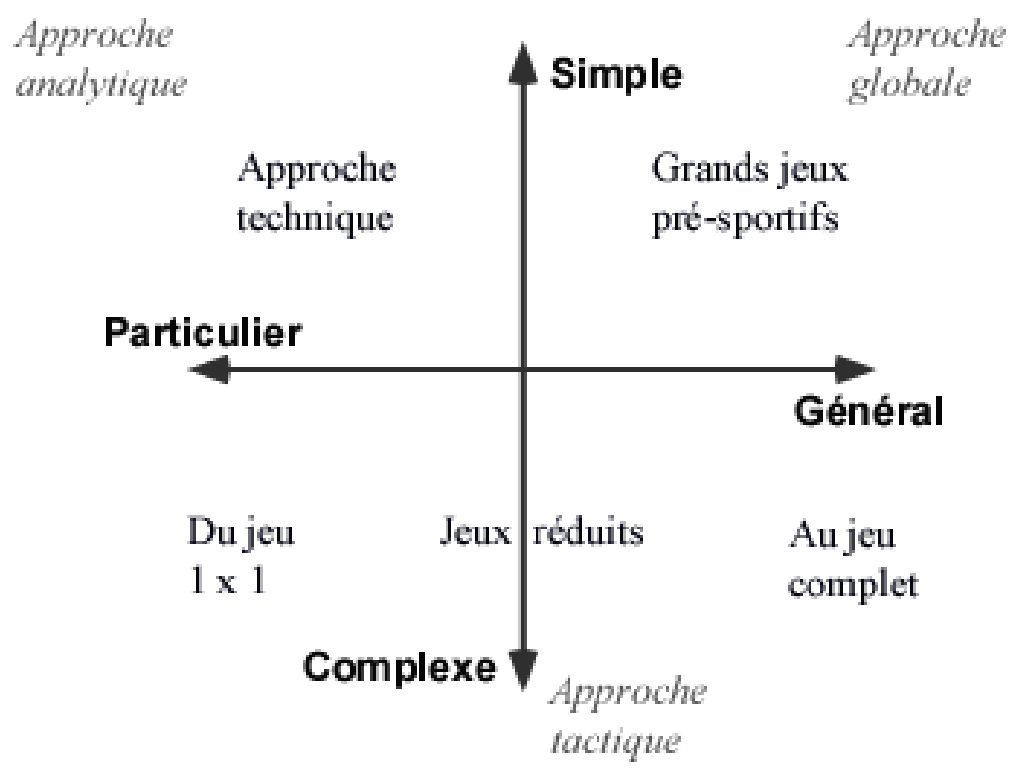

Figure 4. Modélisation des différentes approches d'enseignement des jeux et sports collectifs (adapté de Gréhaigne, 1989).

Une tentative de synthèse pour résumer l'ensemble des approches d'enseignement des JSC pourrait se ramener à ce schéma de principes (Figure 4) permettant une approche macroscopique de la conception dominante employée par un formateur dans l'enseignement des JSC. Cette analyse factorielle n'a pas de valeur normative. Par contre, elle vise simplement à caractériser ce qui est proposé aux élèves, permettant peut-être de mieux comprendre comment se sont développées les études sur ces thèmes.

En développant cette idée d'approche tactique, Gréhaigne $(1988,1989)$ constate dans sa thèse que, dans l'apprentissage classique des sports collectifs, on cherche avant tout à enseigner aux élèves des gestes techniques et à imposer de l'ordre sur le terrain, sous forme de répartition formelle. Pourtant, l'opposition de l'adversaire dans un match engendre de l'imprévu et la nécessité constante de s'adapter aux contraintes issues de l'affrontement. Un match n'est que très rarement la simple application de combinaisons tactiques apprises à l'entraînement. Ainsi, dans le jeu, on ne peut bien souvent envisager que des probabilités d'évolution des configurations de l'attaque et de la défense. De là l'importance d'activités heuristiques permettant de traiter plus vite les problèmes posés par l'interaction spécifique des deux équipes. II convient de distinguer, dans l'utilisation des savoirs, quelle est l'activité du joueur en match et quelle est celle du joueur dans les jeux réduits. En situation de jeu complet, l'élève mobilise toutes ses ressources disponibles (perceptives, attentionnelles, décisionnelles, émotionnelles, énergétiques, motrices...) dans des conditions de pression temporelle qui le conduisent, dans la plupart des cas, à 


\section{eJRIEPS 35 avril 2015}

gérer ou à anticiper les actions des adversaires. C'est à lui de prévoir les déplacements et actions que l'évolution probable du jeu appelle de sa part.

En situation de jeux à effectif réduit, l'activité du joueur relève toutefois d'une autre logique. D'une part, la contrainte temporelle peut être réduite, voire supprimée. D'autre part, il s'agit pour lui de construire des savoirs, compétences perceptives, décisionnelles et motrices, par une succession d'essais, en vue de transformer et d'optimiser ses réponses motrices. L'élève peut ainsi fixer son attention sur un point précis du jeu et explorer l'ensemble des connaissances et leurs variables que la situation a permis d'identifier. La notion centrale d'opposition dans le jeu amène donc à considérer les deux équipes comme des systèmes organisés en interaction. Les caractéristiques structurelles de ces systèmes consistent en un programme modifiable en fonction de l'expérience acquise et leur principale propriété fonctionnelle est l'apprentissage. Les conditions de fonctionnement de ces systèmes en sport collectif font qu'ils ont à gérer avant tout du désordre tout en préservant un certain ordre permettant de décider dans un environnement non complètement prévisible a priori.

En poursuivant les propositions de la pédagogie des modèles de décision tactique, Gréhaigne (1997) ainsi que Gréhaigne, Godbout et Mahut, (1999) proposent un modèle d'apprentissage basé d'abord sur le jeu et la tactique mise en œuvre. La figure 5 propose un schéma construit sous forme spiralaire qui souligne à la fois la continuité des apprentissages et le caractère évolutif et non cyclique des opérations qui permettent ces apprentissages. Les formes et les règles adoptées dans les différents jeux réduits jalonnent et ponctuent le processus permettant l'évolution des élèves. 


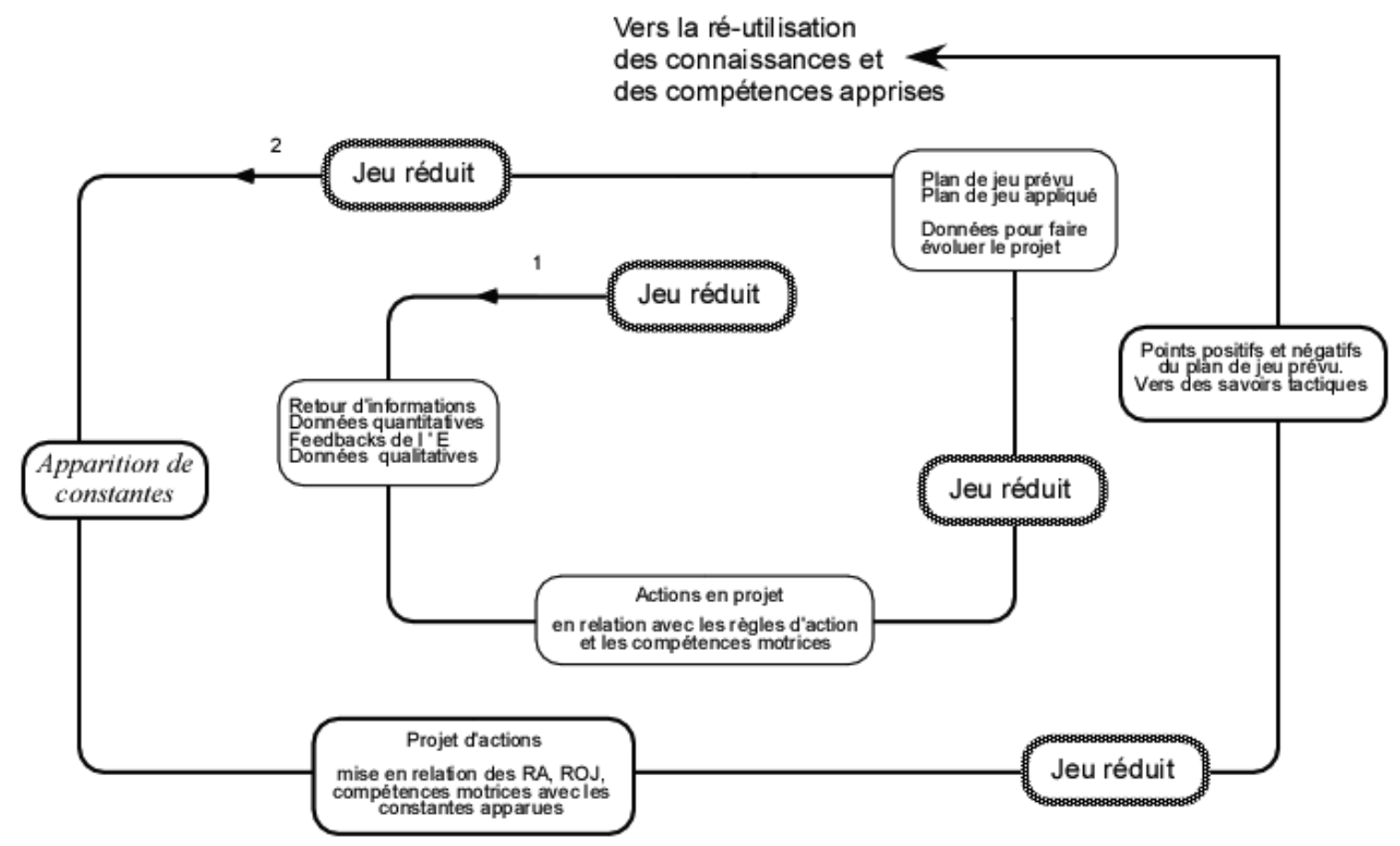

Figure 5. Modèle de décision tactique ou en anglais Tactical Decision Learning Model (TDLM) (Gréhaigne \& Godbout, 1998).

Ainsi, dans ce modèle, les élèves sont mis, dès l'initiation, en activité dans des jeux réduits. Après une observation appropriée, des retours d'informations de natures différentes peuvent compléter les évaluations intrinsèques de chaque joueur. En fonction de ces données et en créant des périodes de discussions entre les joueurs après les séquences de jeu (débats d'idées), chaque équipe met au point un premier projet d'action, qui sera ensuite essayé dans le jeu. Après la séquence de jeu, de nouvelles observations permettront d'apprécier l'efficacité des actions en projet, entraînant d'autres propositions pour une évolution constante du plan de jeu. Après une nouvelle séquence de jeu, les élèves peuvent percevoir l'émergence des constantes de divers aspects de la rencontre. Cela peut conduire à l'élaboration d'une nouvelle action en projet avec la mise en place de liens entre les règles d'action, les règles d'organisation du jeu et les compétences motrices. Après avoir testé son nouveau projet d'action, l'équipe peut utiliser les résultats des observations pour apprécier les aspects positifs et négatifs de leur plan de jeu. Donc, tous les joueurs construisent progressivement des connaissances tactiques. Une fois stabilisés, ces éléments d'apprentissage peuvent être complexifiés ou réutilisés dans un autre sport collectif afin d'engager un processus de généralisation. Aussi, dans ce modèle, on peut repérer trois types de situations d'enseignement / apprentissage :

- les situations d'action où les élèves sont en activité motrice ; 


\section{eJRIEPS 35 avril 2015}

- les situations d'observation ;

- les situations de "débat d'idées" qui sont des situations dans lesquelles les élèves s'expriment et échangent à propos du jeu

Le débat d'idées est une pièce centrale d'une conception sémio-constructiviste (Wallian \& Gréhaigne, 2004) voire socio-sémio-constructiviste de l'apprentissage des sports collectifs. II consiste en une discussion entre les joueurs après une séquence jouée. Cette discussion est basée sur un retour d'informations chiffrées à propos de la performance des joueurs et est destinée à faire évoluer ou non les actions en projet de l'équipe en revenant sur le score, sur la stratégie prévue et en analysant la tactique appliquée lors de la séquence.

\section{D'autres recherches et controverses sur l'approche tactique}

Plusieurs chercheurs et professeurs d'EPS ont soumis des craintes plus ou moins fondées par rapport à l'utilisation d'une approche dite tactique sur les différents aspects de l'enseignement de l'éducation physique chez les élèves.

7.1. D'autres recherches sur l'efficacité comparée de l'enseignement des JSC Ainsi, Chen, Martin, Sun et Ennis (2007) ont tenté de comparer les deux approches (techniques et tactiques) en regard de leurs effets sur la condition physique (fitness) des élèves. L'objet était de vérifier si l'approche constructiviste en EPS, qui semble moins axée sur l'implication motrice des élèves, peut induire une quelconque diminution de la condition physique ou une détérioration des ressources des participants. En effet, certains spécialistes pouvaient prétendre que le temps d'engagement moteur et le temps de pratique des participants peuvent être diminués en raison de la durée des débats d'idées ou de l'efficacité des méthodes ou activités d'apprentissage utilisées dans une approche constructiviste comparativement aux approches traditionnelles. Les résultats de cette étude ont démontré initialement que le niveau de dépense énergétique des élèves était considéré de modéré à faible, peu importe les approches utilisées en EPS. Par ailleurs, les mesures des connaissances acquises par les participants dans une approche constructiviste ont été significativement supérieures aux autres approches pour des élèves de 4ième à la 6ième année (entre 9 et 11 ans). Au final, bien que quelques différences semblent exister entre les deux approches selon le type d'activité enseignée dans les cours, il ne semble pas y avoir une différence significative au niveau de l'effet d'une approche ou d'une autre sur la condition physique des élèves. Par ailleurs, les auteurs soulignent qu'au niveau motivationnel, des différences importantes favorisant une 


\section{eJRIEPS 35 avril 2015}

approche constructiviste ont été constatées. Deux explications ressortent de ces travaux : les élèves se sentent davantage impliqués dans l'organisation des activités et ils considèrent que ce à quoi ils jouent ressemble davantage au jeu réel

Dans le deuxième type d'études qui compare l'efficacité de l'approche technique à celle fondée sur le jeu, deux enjeux sont pris en compte :

1) il importe de savoir comment préparer la séance pour améliorer le sens de l'apprentissage en EPS et déterminer comment se construisent les savoirs (Azzarito \& Ennis, 2003 ; Azzarito, Solmon, \& Afeman, 2003 ; Brooker, Kirk, Braiuka, \& Bransgrove, 2000 ; Pissanos \& Allison, 1993 ; Rovegno, 1998);

2) il importe de comprendre comment l'apprenant développe et construit la tactique en cours d'EPS (Rovegno, Nevett, \& Barbiarz, 2001).

Les populations étudiées s'échelonnent généralement des enfants de CM1 (9-10 ans) jusqu'à l'étudiant en formation et à l'expert enseignant d'EPS. Les études comparant l'efficacité qui ont été répertoriées dans la présente étude utilisent pour la plupart des protocoles quasi-expérimentaux avec des tests cognitifs liés à un sport, y compris des questions sur les connaissances tactiques. Ces études utilisent également des tests standardisés qui mesurent les niveaux d'habiletés motrices. Des grilles d'observation sont aussi utilisées pour mesurer la pertinence des prises de décision. La question au centre de ces mesures est de savoir si les connaissances et les compétences motrices en jeu sont modifiées par la participation à l'une ou l'autre des approches. Un questionnaire mesurant le niveau d'appréciation des participants vient généralement compléter les mesures effectuées pendant l'étude afin de déterminer dans quelle mesure les apprenants ont apprécié leur expérience avec cette approche. Par ailleurs, pour les études ayant pour objectif la description heuristique des faits, le protocole de recherche utilisé est principalement de type qualitatif, comme l'observation participante et l'observation clinique, l'entretien et le journal réflexif de l'enseignant, ainsi que l'analyse des fiches de préparation des leçons.

$\mathrm{Au}$ final, les résultats concernant les études qui ont tenté de comparer les niveaux d'efficacité des deux approches sont contrastés. Pour Griffin, Oslin et Mitchell (1995), Mitchell, Griffin et Oslin (1995) et Turner et Martinek (1992), il n'y a pas de différence significative en termes d'efficacité entre les deux approches avec des cycles courts (5 à 6 leçons). Cependant, l'étude de Turner et Martinek (1999) démontre que les élèves ayant suivi l'approche tactique présentent une meilleure performance du jeu que dans l'approche technique. Dans l'étude de Rink-et al. (1996), les résultats démontrent que les élèves 


\section{eJRIEPS 35 avril 2015}

ayant suivi une approche tactique obtiennent un meilleur score dans le test de connaissances tactiques que ceux ayant suivi une autre approche. Par ailleurs, Wright, Mc Neill, Fry et Wang (2005) établissent que le niveau d'exécution des compétences motrices est meilleur chez les élèves ayant suivi un enseignement tactique que celui des autres élèves.

La variété de ces résultats pourrait s'expliquer par des causes différentes : la durée du cycle d'enseignement est variée d'une étude à l'autre; l'âge de la population diffère également entre les études; les contenus d'enseignement des sports sont différents ; l'efficacité de l'intervention du professeur d'EPS n'a pas été mesurée et comparée aux résultats obtenus, etc. De plus, plusieurs mesures ont été faites de manière isolée et n'ont pas considéré de manière systématique les différentes interactions pouvant exister entre les variables comme le professeur d'EPS, ses compétences, sa compréhension de l'approche, les activités d'apprentissage, les conditions dans lesquelles ont été implantées ces approches, les expériences passées des élèves, etc. Peu importe l'approche utilisée, la question de la préparation du milieu aboutit nécessairement à la qualité et la pertinence de l'intervention de l'enseignant. Cet enseignant doit avoir une connaissance pédagogique suffisante de l'approche en elle-même, des contenus d'enseignement et des compétences d'enseignement associées à l'approche utilisée (PCK ; pedagogical content knowledge) (Amade-Escot, 2000; Rovegno, 1994 ; Siedentop, 2002) en plus de la clarté des attentes et apprentissages visés. Sa compréhension du rapport entre les structures des contenus d'activités doit être profonde et élargie. II semble donc évident qu'il faut partir du point de vue de l'élève pour améliorer le développement des connaissances de l'enseignant. De même, il est nécessaire de préparer adéquatement le milieu afin qu'il favorise l'apprentissage de la coopération sociale chez l'enfant.

Pour ce qui concerne le développement et la construction des habiletés tactiques, les résultats de Rovegno et al. (2001) montrent que l'habileté motrice de la passe et la tactique de l'appel de balle sont hautement situées dans un contexte dynamique. Quand les enfants jouent de cette façon, les défenseurs ont des tâches contraintes, c'est à dire qu'ils prennent davantage conscience des tentatives de l'adversaire de faire progresser le jeu. Cela permet ainsi d'augmenter l'authenticité du jeu. Les élèves s'impliquent ainsi dans un contexte social qui autorise un apprentissage signifiant au regard des contenus enseignés.

\subsection{L'interactionnisme discursif}




\section{eJRIEPS 35 avril 2015}

Chang (2006) a exploré de son côté comment des élèves taïwanais de CM2 apprennent en basket-ball avec une approche sémio-constructiviste, soit une approche «mettant l'élève au cœur des processus d'appropriation/construction des savoirs dans et par l'action » Makram (2012). Les trois aspects de l'étude se sont focalisés sur 1) l'analyse des matchs, 2) les échanges verbaux dans le débat d'idées et 3) un test vidéo de l'interprétation du jeu. Deux classes comparables ont été divisées en un groupe expérimental suivant une approche constructiviste (17 filles ; 15 garçons), et en un groupe témoin suivant un enseignement techno-centré (16 filles ; 16 garçons). Autant le jeu de basket-ball que les échanges verbaux entre les participants ont été enregistrés durant un cycle complet d'apprentissage (12h de pratique effective). La dynamique du jeu a été, elle, analysée par une observation systématique, alors que les débats entre élèves et les interprétations du test vidéo étaient transcrits, traduits et traités par une analyse du discours.

Trois types de résultats sont ressortis de cette étude:

1) l'approche sémio-constructiviste implique la mobilisation d'un système complexe de ressources (techniques, interprétatives, décisionnelles, sémiotiques et sociales) ;

2) l'évolution du rapport de forces pendant le jeu semble faire progresser l'organisation du jeu et suscite la prise de décision tactique ;

3) la relation entre le langage et l'action s'améliore sous certaines conditions pendant l'apprentissage en basket-ball.

En somme, si l'efficacité à court terme du modèle technique a été confirmée dans de nombreuses recherches (French et al., 1991; Sweeting \& Rink, 1999), les études qui ont mesuré les effets à long terme prouvent que le modèle tactique démontre une plus grande efficacité pour la performance en jeu que le modèle technique après 6 à 8 leçons. Différentes études, comme celles de Chang (2006), Zerai (2006), Berchebru (2007), et Gréhaigne, Richard et Griffin (2005), permettent de constater que la période de 6 à 8 leçons est la période nécessaire pour que les apprentissages en jeu commencent à évoluer de façon significative. Avant cette période, des réponses nouvelles ne font juste qu'émerger des élèves confrontés aux problèmes vécus en situation de jeu. Du temps, de la pratique et de la réflexion sont essentiels pour que ces réponses deviennent des réponses stabilisées et de qualité et qui seront disponibles comme ressources pour l'élève dans les cycles à venir.

7.3. Le concept de «Team Cognition » 


\section{eJRIEPS 35 avril 2015}

Dernier point, nous n'avons pas traité des analyses fondées sur l'approche par la « Team cognition »(Cooke, Gorman, \& Winner, 2007 ; Bourbousson \& Seve, 2010), car cette approche, importée de la psychologie (cognition appliquée), a malheureusement tendance à oublier les adversaires... au profit d'une centration exclusive sur le groupe. De plus, l'approche semble parfois confondre la notion d'équipe avec celle de couple. Pourtant, dans sa thèse, Gréhaigne (1989) écrivait déjà que dans les JSC, la possession ou non du ballon détermine des statuts différenciés : porteur de balle, partenaire du porteur de balle, défenseur sur le porteur de balle, défenseur sur le partenaire du porteur de balle. Aussi, il apparaît difficile de considérer la situation de $2 \times 2$ en jeu réel comme une véritable situation de JSC car cela remettrait en cause la définition, généralement admise, des JSC où un groupe affronte un autre groupe, "une modalité originale d'exploitation de la dynamique des groupes restreints poursuivant un objectif commun » ( Mérand, 1977, p. 12). Or, Anzieu et Martin (1968, p 30) définissent « un groupe restreint comme étant la réunion de trois à quinze personnes ». Gréhaigne (1989) proposait alors, que le 3 x 3 , c'est-à-dire un jeu qui réunit porteur de balle, réceptionneur éventuel 1, réceptionneur éventuel 2, défenseur 1 , défenseur 2 , défenseur 3 , soit la structure minimale pour qu'il y ait réellement sport collectif. Ce $3 \times 3$ permet de passer de choix binaires (je passe ou je conserve la balle) à des choix multiples : je garde ou je passe à J2 ou je passe à J3, conservant ainsi la complexité minimale pour que nous puissions parler de sports collectifs. En dessous de cette structure, nous sommes dans des situations de jeu présport collectif qui ne rendent pas compte de cette complexité minimale du jeu. Alors, le $2 \mathrm{x}$

2 représente une tâche partielle de jeu à résoudre dans des cas bien précis et non pas "la plus petite micro-société des J.S.C. qui réalise un affrontement équitable, structure originelle, réelle et profonde de l'ensemble des J.S.C» (Menaut (1982), p 40).

Enfin, il est patent que la notion d'articulation du cours d'action entre joueurs partenaires, si elle éclaire certaines dimensions de l'action située, ne permet pas de rendre compte de la logique tactique collective au sein des relations d'opposition.

\section{De l'enseignement des jeux pour la compréhension à l'apprentissage du jeu à travers la compréhension : perspectives ouvertes par les recherches actuelles}

Kirk et MacPhail (2002) notent que la notion de performance, située dans l'approche TGFU, fournit une façon de comprendre la relation entre la forme de jeu d'une part, et la conception préalable et alternative du joueur au jeu d'autre part (voir aussi Gréhaigne \& Godbout, 1998). II est ainsi important pour les élèves de saisir le sens réel du jeu selon 


\section{eJRIEPS 35 avril 2015}

l'activité pratiquée (Houdé, 1992). La signification pour l'élève se réfère à sa capacité à formaliser de manière explicite ou implicite ses observations en fonction des instructions et de la tâche, avant d'être en mesure de les traiter.

\section{Système enseignement / apprentissage}

\section{Enseigner les jeux pour la compréhension}

Jeu à effectif réduit avec un problème caché

\section{Contenu d'enseignement situé} (identification des configurations)

Organiser le débat d'idées et l'observation

Enseigner des éléments tactiques

\section{Apprendre les jeux à travers} la compréhension

Jouer / Observer / Percevoir les configurations du jeu

Débat d'idées dans lequel plusieurs solutions sont valables et validées

Verbalisations vers des actions en projet

Vérification en jeu / Transformation

Figure 6. Processus impliqués dans Teaching Games for Understanding - TGfU (Bunker \& Thorpe, 1982 ; Butler, Griffin, Lombardo, \& Nastasi, (2003); Hopper, Butler \& Storey. (2009) et Learning Games through Understanding - LGtU (Gréhaigne, Caty, \& Godbout, 2010).

En ce qui concerne le rapport entre l'enseignement et l'apprentissage des jeux à l'école, la figure 6 illustre en parallèle les caractéristiques d'un enseignement des jeux pour la compréhension (TGFU) organisé par l'enseignant et de l'autre celles d'un apprentissage des jeux au travers de la compréhension (LGtU) basé sur l'activité de l'élève. L'enseignement des jeux pourla compréhension ne peut pas garantir à lui seul une véritable approche centrée sur l'élève. Le fait de proposer l'apprentissage des jeux à travers la compréhension (Gréhaigne, Zerai, \& Caty, 2009) nous semble bien plus susceptible de créer un environnement d'apprentissage plus authentique, prometteur et de garantir une approche centrée sur les apprenants. En effet, dans TGfU, l'enseignant peut poser des questions parfaitement ou en partie fermées et donner " la piqûre de savoir » ou la solution au moment choisi par lui et non par les élèves. Dans ce type de situation, on peut en revenir très vite à un enseignement centré sur le maître. 


\section{eJRIEPS 35 avril 2015}

Le fait d'insister sur l'apprentissage dans le système enseignement / apprentissage, avec le strict respect du débat entre les élèves, nous semble susceptible de mieux garantir un environnement d'apprentissage authentique et prometteur et de garantir une approche centrée sur les élèves. Une telle approche pédagogique n'est pas destinée à amuser les élèves mais à leur faire appréhender le jeu différemment. Le but sous-jacent essentiel, en droite ligne avec une perspective cognitiviste et constructiviste (Gréhaigne \& Godbout, 1995), est bien de demander aux élèves de découvrir les problèmes à résoudre puis de faire une prévision de jeu afin d'apporter des réponses aux problèmes posés.

Dans une approche socio-sémio-constructiviste de l'enseignement, les apprenants s'engagent activement dans le processus d'apprentissage en EPS. Pour les JSC, les élèves sont amenés à construire leurs compétences tactiques en les adaptant individuellement selon la situation spécifique vécue. Une telle option soutient que les connaissances construites par l'élève sont le résultat de l'interaction entre son activité cognitive et la réalité (Cobb, 1986 ; Gréhaigne \& Godbout, 1998; Piaget, 1974).

Dans une approche d'enseignement considérée comme indirecte, «Apprendre le jeu au travers de la compréhension » devrait être la principale philosophie à développer. L'apprentissage des élèves dans une approche constructiviste les amène donc à tenter de donner un sens à l'entrée de nouvelles réponses en les rapportant à leurs connaissances antérieures et en collaborant à une discussion avec d'autres élèves pour construire une compréhension partagée de la réponse envisagée. Comme il a été déjà mentionné, tout processus de résolution de problèmes d'apprentissage, de même que la construction de connaissances personnelles, nécessite une réflexion de la part des élèves. Cette réflexion se déroule parfois pendant une période qui peut être très variable selon les capacités intellectuelles des élèves et leur vécu sportif. La verbalisation pendant les discussions peut, pour beaucoup d'entre eux, faciliter la réflexion et l'observation, de même que fournir les données de base sur lesquelles réfléchir. Ainsi, les élèves collaborent en agissant comme une communauté d'apprentissage qui construit graduellement sa compréhension partagée du jeu à travers un dialogue et l'expérimentation réelle en situation de jeu.

Enfin, la «transformation » implique non seulement l'apparition de nouvelles réponses à un problème donné, mais aussi une stabilisation de ces réponses. En effet, l'apparition d'une nouvelle réponse chez l'élève ne veut en aucun cas dire qu'elle sera utilisée ultérieurement. Sous la pression temporelle, l'élève peut en revenir à un modèle ancien de lecture du jeu. Des réponses nouvelles seront considérées comme stabilisées si elles répondent à trois critères : la systématicité, la durabilité, la généralisation. Pour ce type 


\section{eJRIEPS 35 avril 2015}

d'approche et particulièrement lors des périodes de discussion, l'enseignant devient une personne ressource pour les élèves. Sa principale tâche est de faciliter d'une part un dialogue respectueux entre les participants, et d'autre part, la réflexion des élèves par certaines relances lorsque les discussions ne mènent à rien. Ce rôle d'enseignant personne ressource - diffère totalement des approches traditionnelles d'enseignement des sports où il est davantage considéré comme un transmetteur de savoir.

Cette culture différente de «l'enseignement » peut, au départ, insécuriser les jeunes collègues en formation. Mais rapidement ce changement dans le rôle de l'enseignant motive au contraire les élèves en les rendant plus impliqués dans le déroulement de la séance. Enfin, permettre aux élèves de profiter des expériences menées et de construire des connaissances nouvelles suppose de laisser une place à du tâtonnement, à de la découverte guidée et de faire toute sa place à une conception inductive de la transmission des connaissances.

\section{Conclusion}

Pour Good (1996), la perspective de l'enseignement / apprentissage au travers de la compréhension est à l'heure actuelle la plus puissante tradition intégratrice susceptible d'influencer les pratiques des enseignants dans une classe. Si TGfU et les débats de la recherche anglo-saxonne ont permis de populariser à l'échelle mondiale la question de l'enseignement de la tactique en sport collectif, il reste que leur oubli ou leur méconnaissance des travaux des aires non anglophones limite la portée pratique de ceuxci.

Certes, le débat d'idées donne la possibilité aux élèves de partager des expériences collectives dans le cadre d'un rapport de forces en regard des configurations momentanées du jeu apparues. Mais, offrir aux élèves la meilleure éducation physique possible implique bien évidemment de nombreux autres facteurs. L'un des plus importants est de savoir comment les enseignants enseignent et surtout comment les élèves apprennent. Les chercheurs ont besoin d'approfondir les voies d'apprentissage et de décrire, chez les élèves, les processus cognitifs et la construction de l'expérience collective qui se produisent pendant le jeu. En faisant cela, on pourra mieux comprendre les mécanismes qui influencent les relations entre l'acquisition des connaissances et les performances motrices. Un environnement d'apprentissage efficace crée nécessairement des conditions d'enseignement efficientes. Pour atteindre ce but, il est essentiel que les enseignants deviennent des praticiens réflexifs, suffisamment informés des avancées de 


\section{eJRIEPS 35 avril 2015}

la recherche et, peut-être, plus attentifs aux besoins des élèves qu'à la matière à enseigner.

Enfin, Didier Caty, un de nos collègues du lycée de Gray, témoignait (Caty, Zerai, \& Gréhaigne, 2009) :

au-delà des résultats chiffrés et des tests, parfois peu significatifs pour le lecteur, ce descriptif ne peut communiquer tous les évènements, toutes les joies et tous les débats qui ont ponctué ce cycle de travail. Comment retranscrire les rares absences ? Comment dire au lecteur que certains élèves n'avaient pas cours depuis le matin et restaient pour participer? Comment expliquer que certaines ont joué alors qu'elles étaient physiquement diminuées? La dynamique créée autour d'un cycle essentiellement basé sur le jeu n'a sans doute pas permis aux plus faibles de se transformer radicalement. Toutefois, l'originalité de ce type de travail a suscité beaucoup de plaisir chez les élèves et donné du sens aux leçons. (p. 227)

Tout est dit !

Les auteurs remercient les deux experts pour leurs remarques et conseils ainsi que Chantal Amade-Escot et Paul Godbout pour leur relecture attentive d'une version préliminaire de ce texte.

\section{Bibliographie}

AEEPS (Amicale des Enseignants d'Éducation Physique) (1984). Sport co en milieu scolaire. Paris : Amicale ENSEPS.

Amicale des Anciens Élèves de l'ENSEPS (1966). Des colloques de Vichy 1964 - 1965. Éducation Physique et Sport, 78, 19-73.

Amade-Escot, C. (1997). "Sport Education" : Une orientation curriculaire nord-américaine. Contre Pied, 1, 35-39.

Amade-Escot, C. (2000). The contribution of two research programs on teaching content: "Pedagogical Content Knowledge" and "Didactics of Physical Education". Journal of Teaching in Physical Education, 20, 78-101.

Anderson, J. R. (1982). Acquisition of cognitive skill. Psychological Review, 89, 369-406.

Anzieu, D., \& Martin, J. Y. (1968). La dynamique des groupes restreints. Paris : PUF.

Azzarito, L., \& Ennis, C. D. (2003). A sense of connection: Toward social constructivist physical education [Abstract]. Sport, Education and Society, 8, 179-198. 


\section{eJRIEPS 35 avril 2015}

Azzarito, L., Solmon, M. A., \& Afeman, H. (2003, April). Investigating a constructivist approach in physical education: Bridging theory and practice. Paper presented at the annual meeting of the American Alliance for Health, Physical Education, Recreation and Dance, Philadelphia, PA.

Barth, B. (1978). Zur technisch-taktischer grundausbilding im fetchen. Theorie und Praxis der Körperkultur, 27, 288-293.

Barth, B. (1980). Probleme einer theorie und methodik des strategie und taktik des wettkampfes im sport. Theorie und Praxis der Körperkultur, 29, 374-384.

Berchebru, M. (2007). Didactique de l'enseignement des sports collectifs. Quand l'utilisation de technologies multimédia et de la verbalisation améliore l'enseignement des sports collectifs (Mémoire de Mastère non publié). Besançon : Université de Franche-Comté.

Bourbousson, J., \& Seve, C. (2010). Construction / déconstruction du référentiel commun d'une équipe de basket-ball au cours d'un match. eJournal de la Recherche sur I'Intervention en Education Physique et Sportive, 20, 5-21.

Bouthier, D. (1984). Sports collectifs : contribution à l'analyse de l'activité et éléments pour une formation tactique essentielle. L'exemple du rugby (Mémoire non publié). Paris : INSEP.

Bouthier, D. (1986). Comparaison expérimentale des effets de différents modèles didactiques des sports collectifs. Dans E.P.S contenus et didactique (pp. 85-89). Paris : SNEP.

Bouthier, D. (1988). Les conditions cognitives de la formation d'actions sportives collectives (Thèse non publiée). Paris : Université Paris V. EPHE.

Brooker, R., Kirk, D., Braiuka, S., \& Bransgrove, A. (2000). Implementing a game sense approach to teaching junior high school basketball in a naturalistic setting. European Physical Education Review, 6, 7-26.

Bunker, D., \& Thorpe, R. (1982). A model for the teaching of games in secondary schools. Bulletin of Physical Education, 18 (1), 5-8.

Butler, J., Griffin, L., Lombardo, B., \& Nastasi, R. (2003). Teaching for understanding in physical education and sport. Oxon Hill: AAPHERD Publications.

Caron, J., \& Pelchat, C. (1974). Le Hockey, sport collectif : modèle empirique ou théorique. Mouvement, 9 (1), 33-46.

Caron, J., \& Pelchat, C. (1975). Apprentissage des sports collectifs, hockey et basket. Québec : Presses de l'Université du Québec. 


\section{eJRIEPS 35 avril 2015}

Caty, D., Zerai, Z., \& Gréhaigne. J.-F. (2009). Un cycle de handball au lycée de Gray et de Thala. Dans J. F. Gréhaigne (Ed.) Autour du temps Espaces, apprentissages, projets dans les sports collectifs (pp. 189-219). Besançon: Presses de l'Université de Franche-Comté.

Chang, C. W. (2006). Vers une approche constructiviste de l'enseignement du basket-ball à Taïwan (Thèse non publiée). Taïpei : National Taïwan Normal University.

Chen, A., Martin, R., Sun, H., \& Ennis, C. D. (2007). Is physical activity at risk in constructivist physical education? Research Quarterly for Exercise and Sport, 78, 500-509.

Chi, M. T., \& Rees, E. T. (1983). A learning framework for development. Dans M. T. H. Chi (Ed.), Contributions in human development, Vol. 9, (pp. 71-107). Basel: Karger.

Cobb, P. (1986) Making mathematics: Children's learning and the constructivist tradition. Harvard Educational Review, 56, 301-306.

Cooke, N. J., Gorman, J. C., \& Winner, J. (2007). Team cognition. Dans F., Durso, R. Nickerson, S. Dumais, S. Lewandowsky, \& T. Perfect (Eds.), Handbook of applied cognition (pp. 239-268). New York: Wiley.

David, B. (1984). Initiation précoce au rugby : étude comparée de deux méthodes d'apprentissage (Mémoire de Maîtrise en STAPS non publié). Paris : Université Paris V.

Deleplace, R. (1966). Le rugby. Paris : Colin - Bourrelier.

Deleplace, R. (1979). Rugby de mouvement-Rugby total. Paris: Éducation Physique et Sport.

Den Duyn, N. (1997). Game Sense, It's time to play. Sports Coach, 19 (4), 9-11.

Diaz, J. C. (1983). Problèmes posés par l'apprentissage du rugby chez de jeunes enfants. L'exemple d'une situation de un contre un (Mémoire de Maîtrise en STAPS non publié). Paris : Université Paris V.

Dyotte, G., \& Ruel, A. (1976). Techniques du hockey en U.R.S.S./ Stratégies du "système russe". Montréal, Québec : Éditions De L'Homme.

French, K. E., \& Thomas, J. R. (1987). The relation of knowledge development to children's basketball performance. Journal of Sport Psychology, 9, 15-32.

French, K. E., Rink, J. E., Rikard, L., Mays, A., Lynn, S. \& Werner, P. (1991). The effects of practice progressions on learning two volleyball skills. Journal of Teaching in Physical Education, 10, 261-274. 


\section{eJRIEPS 35 avril 2015}

Good, T. (1996). Teaching effects and teacher evaluation. Dans J. Sikula, T. J. Buttery, \& E. Guyton (Eds.), Handbook of research on teacher education (2nd edition) (pp. 617-665). New-York : Simon \& Schuster Macmillan.

Gréhaigne, J.-F. (1988). Game systems in soccer. Dans T. Reilly, A. Lees, K. Davids, \& W. J. Murphy (Eds.), Science and Football (pp. 316-321). Londres : E. \& F.N. SPON. Gréhaigne, J.-F. (1989). Football de mouvement. Vers une approche systémique du jeu (Thèse de Doctorat non publiée). Dijon, France : Université de Bourgogne.

Gréhaigne, J.-F. (1994). Analyse comparative de deux types d'enseignement des sports collectifs : approche centrée sur la technique et approche centrée sur le jeu (Rapport de Recherche non publié). Besançon: UFR-STAPS, Université de Franche-Comté.

Gréhaigne, J.-F. (1997). Modélisation du jeu de football et traitement didactique des jeux sportifs collectifs (Habilitation à diriger les recherches non publiée). Orsay, France : Université de Paris-Sud Orsay.

Gréhaigne, J.F., \& Godbout, P. (1995). Tactical knowledge in team sports from a constructivist and cognitivist perspective. Quest, 47, 490-505.

Gréhaigne, J.-F., \& Godbout, P. (1998). Observation, critical thinking and transformation: Three key elements for a constructivist perspective of the learning process in team sport. Dans R. S. Feingold, C. R. Rees, G. T. Barrette, L. Fiorentino, S. Virgilio, \& E. Kowalski (Eds.), Education for life (pp. 109-118). Garden City (NY): Adelphi University.

Gréhaigne, J.F., Caty, D., \& Godbout, P. (2010). Modelling ball circulation in invasion team sports: a way to promote learning games through understanding. Physical Education and Sport Pedagogy, 15, 257-270.

Gréhaigne, J.-F., Godbout, P., \& Mahut, N. (1999). L'enseignement des jeux par la compréhension: une revue de questions. STAPS , 48, 81-93.

Gréhaigne, J.-F., Richard, J.-F., \& Griffin, L. (2005). Teaching and learning team sports and games. New York: Routledge, Taylor \& Francis Group.

Gréhaigne. J.-F., Zerai, Z., \& Caty, D, \& (2009). Prototypic Configurations of Play in Handball in Physical Education: A Strategy to Promote Student Understanding in Team Sports. In T. Hopper, J. Butler \& B. Storey (Eds.), Teaching game for understanding : Simply good pedagogy: Understanding a complex challenge (pp. 53-64). Ottawa : PHE Canada. 


\section{eJRIEPS 35 avril 2015}

Griffin, L. L., Oslin, J. L., \& Mitchell, S. A. (1995). An analysis of two instructional approaches to teaching net games [Abstract]. Research Quarterly for Exercise and Sport, 66, 64.

Hopper, T., Butler, J., \& Storey, B. (2009). TGfU...Simply Good Pedagogy: Understanding a complex challenge. Ottawa: PHE Canada.

Houdé, O. (1992). Catégorisation et développement cognitif. Paris: PUF.

Kirk, D., \& MacPhail, A. (2002). Teaching Games for Understanding and situated learning: Rethinking the Bunker and Thorpe model. Journal of Teaching in Physical Education, 21, 177-192.

Lawton, J. (1989). Comparison of two teaching methods in games. Bulletin of Physical Education, 25 (1) 35-38.

Lenzen, B., Poussin, B., Deriaz, D., Dénervaud, H., \& Cordoba, A. (2010). Conceptions de futurs enseignants généralistes et D'EPS à propos de l'évaluation dans les jeux collectifs. Formation et Pratiques d'Enseignement en Questions, 11, 91-113. http://www.revuedeshep.ch/site-fpeq/Site_FPEQ/11_files/06_lenzen.pdf

Leontiev, A. N. (1957). L'apprentissage comme problème de psychologie. Voprossy psikhologuii, 1, 11-12 (en langue russe).

Leontiev, A. N. (1972). Le développement du psychisme. Paris : Éditions sociales.

Mac Pherson, S. L., \& French, K. E. (1991). Changes in cognitive strategies and motor skill in tennis. Journal of Sport and Exercise Psychology, 13, 26-41.

Mahlo, F. (1965). Theoretische problem der taktischen : Ausbildung in den sportspielen. In Theorie und praxis der körperkultur, 14 (9), 809-816 ; (1965) - (11), 967-979 ; (1965) - (12), 1075-1082 ; (1966) Theorie und praxis der körperkultur, 15, (1), 2229 ; (1966) - (2) 10-17 ; 1966) - (3) 228-238).

Mahlo, F. (1969). Acte tactique en jeu. Paris : Vigot.

Makram, Z. (2012). Approche sémio-constructiviste et apprentissage de jeu en Football. Sarrebruck : Éditions universitaires européennes.

Marsenach, J., \& Druenne, F. (1974). Volley-ball. Mémento CPS FSGT. Paris : Armand Colin-Bourrelier.

Menaut, A. (1982). Contribution à une approche théorique des jeux sportifs collectifs (Thèse non publiée de doctorat d'état en Sciences de l'Education). Bordeaux : Université de Bordeaux 2.

Méot, E. (1982). Vers la simulation du jeu de football par ordinateur (Thèse d'Université non publiée). Lyon : Université de Lyon 1. 


\section{eJRIEPS 35 avril 2015}

Méot, E., \& Plumereau, J. (1979). Quelques éléments pour définir des objectifs. Éducation Physique et Sport, 159, 59-64.

Mérand, R. (1977). L'éducateur face à la haute performance. Paris : Editions Sport et plein air.

Mérand, R. (1989). La rénovation des contenus d'enseignement : Jeux sportifs collectifs au collège. Revue Française de Pédagogie, 89, 11-14.

Ministère de la Jeunesse et des Sports; Ministère de l'Education Nationale (1967). Instructions officielles aux professeurs et maîtres d'éducation physique et sportive. Paris : BOEN 41 (Circulaire du 19 octobre 1967).

Mitchell, S. A., Griffin, L. L., \& Oslin, J. L. (1995). The effects of two instructional approaches on game performance. Pedagogy in Practice: Teaching and Coaching in Physical Education and Sports, 1(1), 36-48.

Piaget, P. (1974). Réussir et comprendre. Paris : PUF.

Pissanos, B. W., \& Allison, P. C. (1993). Students' constructs of elementary school physical education. Research Quarterly for Exercise and Sport, 64, 425-435.

Reitchess, S. (1983). Problèmes posés par l'apprentissage d'actions collectives en rugby en utilisant une pédagogie des choix tactiques avec de jeunes enfants (Mémoire non publié de Maîtrise en STAPS). Paris : Université Paris V.

Riley, M., \& Roberton, M. A. (1981). Developing skillful game players consistency between beliefs and practice. Motor skills: Theory into practice, 5, 123-133.

Rink, J., French, K. \& Werner, P. (1991, January). Tactical awareness as the focus for ninth grade badminton. Paper presented at AIESEP World congress, Atlanta, Georgia.

Rink, J. E., French, K. E., \& Tjeerdsma, B. L. (1996). Foundations for the learning and instruction of sport and games. Journal of Teaching in Physical education, 15, 399-417.

Rovegno, I. (1994). Teaching within a curricular zone of safety: School culture and the situated nature of student teachers' pedagogical content knowledge. Research Quarterly for Exercise and Sport, 65, 269-279.

Rovegno, I. (1998). The development of in-service teachers' knowledge of a constructivist approach to physical education: Teaching beyond activities. Research Quarterly for Exercise and Sport, 69, 147-162. 


\section{eJRIEPS 35 avril 2015}

Rovegno, I., Nevett, M., \& Babiarz, M. (2001). Learning and teaching invasion-game tactics in 4 th grade: Introduction and theoretical perspective. Journal of Teaching in Physical Education. 20, 352-369.

Syndicat National de l'Education Physique (1984). L'évaluation en EPS. Paris : SNEP.

Siedentop, D. (1983) Developping teaching skills in physical education. Mountain View, CA : Mayfield.

Siedentop, D. (2002). Content knowledge for physical education. Journal of Teaching in Physical Education. 21, 368-377.

Stein, J.-F. (1981). Sports d'opposition, éléments d'analyse pour une pédagogie des prises de décisions (Mémoire non publié). Paris, INSEP.

Sweeting, T., \& Rink J. E. (1999). Effects of direct instruction and environmentally designed instruction on the process and product characteristics of a fundamental skill. Journal of Teaching in Physical Education, 18, 216-233.

Teodorescu, L. (1965). Principes pour l'étude de la tactique commune aux jeux sportifs collectifs. Revue de la S.I.E.P.E.P.S., 3, 29-40 (texte publié à nouveau dans eJRIEPS 28, 99-117).

Teodorescu, L. (1977). Théorie et méthodologie des jeux sportifs. Paris : Les Editeurs Français Réunis.

Thomas, J. R., French, K. E., \& Humphries, C. A. (1986). Knowledge development and sport skill performance. Directions for motor behavior research. Journal of Sport Psychology, 8, 259-272.

Thomas, J. R., French, K. E., Thomas, K. T., \& Gallagher, J.D. (1988). Children's knowledge development and sport performance. Dans F. L. Smoll, R. A. Magill, \& M. J. Ash (Eds.), Children in sport (p. 179- 202). Champaign, IL : Human Kinetics.

Turner, A.P. (1993, March). A model for working with students with varying knowledge structures. Paper presented at the AAHPERD National Conference, Washington, DC.

Turner, A. P., \& Martinek, T. J. (1992). Analyse comparative de deux modèles d'enseignement des jeux : approche technique et approche centrée sur le jeu (aspect tactique). Département des sciences du sport. Université de Caroline du Nord à Greensboro, USA.

Turner, A. P., \& Martinek, T. J. (1999). An investigation into teaching games for understanding: Effects on skills, knowledge, and game play. Research Quarterly for Exercise and Sport, 70, 286-296. 


\section{eJRIEPS 35 avril 2015}

Vandevelde, M. (2006). Education physique et basket-ball. Robert Mérand : un regard neuf sur l'activité de l'élève. Paris : Syllepse.

Villepreux, P. (1987). Rugby de mouvement et disponibilité du joueur (Mémoire non publié). Paris : INSEP.

Vygotsky, L. (1933). Pensée et langage (traduction de Françoise Sève et avant-propos de Lucien Sève), suivi de "Commentaires sur les remarques critiques de Vygotski » de Jean Piaget, (Collection «Terrains», Éditions Sociales, Paris, 1985) ; Réédition, Paris : La Dispute, 1997.

Wade, A. (1967). The football association guide to training and coaching. London : E. P. Publishing LTD.

Wallian, N., \& Gréhaigne, J.-F. (2004). Vers une approche sémioconstructiviste des apprentissages moteurs. Dans G. Carlier (Ed.), Si l'on parlait du plaisir d'enseigner l'éducation physique (pp. 167-179). Montpellier : AFRAPS.

Wilkinson, W. H. G. (1978). The forming of a theoretical model of sport strategy in professional soccer (Unpublished doctoral dissertation). Liverpool, England: Department of physical education, Liverpool Polytechnic.

Worthington, E. (1974). Teaching soccer skill. Londres : Lepus books.

Wright, S., Mc Neill, M., Fry, J., \& Wang, J. (2005). Teaching teachers to play and teach games. Physical Education and Sport Pedagogy, 1, 61-82.

Zerai, Z. (2006). Comment les filles apprennent-elles en Hand-Ball? Apport de la verbalisation (Mastère non publié en didactique des Activités Physiques et Sportives). Tunis : ISEFC. 